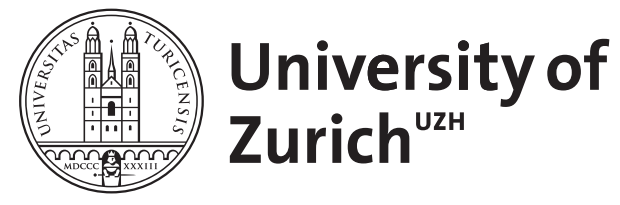

\title{
Regulating Immunity via ADP-Ribosylation: Therapeutic Implications and
} Beyond

\author{
Kunze, Friedrich A ; Hottiger, Michael O
}

DOI: https://doi.org/10.1016/j.it.2018.12.006

Posted at the Zurich Open Repository and Archive, University of Zurich ZORA URL: https://doi.org/10.5167/uzh-168110

Journal Article

Accepted Version

Originally published at:

Kunze, Friedrich A; Hottiger, Michael O (2019). Regulating Immunity via ADP-Ribosylation: Therapeutic Implications and Beyond. Trends in Immunology, 40(2):159-173.

DOI: https://doi.org/10.1016/j.it.2018.12.006 
Regulation of the immune response by ADP-ribosylation: therapeutic implications and beyond

Friedrich A. Kunze ${ }^{\mathrm{a}, \mathrm{b}}$ and Michael O. Hottiger ${ }^{\mathrm{a}, 1}$

${ }^{\mathrm{a}}$ Department of Molecular Mechanisms of Disease, University of Zurich, Winterthurerstrasse 190, 8057 Zurich, Switzerland,

${ }^{\mathrm{b}}$ Molecular Life Science PhD Program of the Life Science Zurich Graduate School, Winterthurerstrasse 190, 8057 Zurich, Switzerland,

${ }^{1}$ Corresponding author: michael.hottiger@dmmd.uzh.ch

\begin{abstract}
Innate immune cells express pattern recognition receptors (PRRs) that recognize pathogenassociated molecular patterns (PAMPs) and endogenous danger-associated molecular patterns (DAMPs). Upon binding PAMPs/DAMPs they initiate an immune response by activating lymphocytes that amplify and modulate signaling cascades to induce an appropriate effector response. Protein ADP-ribosylation has been reported to regulate cell death, the release of DAMPs and inflammatory cytokine expression. Inhibitors of ADP-ribosylation (i.e. PARP inhibitors) have been developed as cancer therapeutics, but have also been reported to dampen inflammation. Here we summarize our current understanding how ADP-ribosylation regulates the different phases of an immune response and the potential clinical translation of pharmacological ADP-ribosylation inhibitors for the therapy of various inflammation-associated diseases.
\end{abstract}

Boxes $=$ green

Glossary $=$ magenta 


\section{ADP-ribosylation: The link between DNA damage and inflammation}

ADP-ribosylation mediated by intracellular mammalian ADP-ribosyltransferases (ARTs, formerly PARPs) - in particular ARTD1 (PARP1) - has first been identified and extensively studied in the context of the DNA damage response and the maintenance of genome stability (Box 1 and references therein). In recent years, the field started to investigate ARTs and their impact on inflammatory gene expression. Inhibitors of ADP-ribosylation (i.e. PARP-inhibitors) gained much attention in cancer therapy but recent findings also suggest anti-inflammatory activity (Box 2). This review provides a comprehensive summary of poly- and mono-ADP-ribosylation during tissue damage-, bacteria- or virus-induced inflammation in mammalian cells. First, we will discuss ADP-ribosylation during the induction of sterile inflammation. Second, we will present ART functions during the different pathogen-associated molecular pattern (PAMP) and endogenous danger-associated molecular pattern (DAMP)-induced phases of an immune response. Finally, we will touch the function of ADPribosylation as an innate anti-viral response and shed some light on the use of PARPi during inflammation. Important mechanistic and basic findings as well as the most recent developments in the field will be presented and discussed.

\section{ADP-ribosylation-induced cell death and the release of danger associated molecular patterns (DAMPs)}

Cell death can be initiated in a regulated manner (i.e. apoptosis or autophagy) or by tissue injury (necrosis). While apoptotic cell death does not induce an inflammatory response, the necrotic cell death does so due to the release of DAMPs or alarmins ${ }^{1}$. The constant exposure of cells to stressors, such as UV light, reactive oxygen species (ROS) or alkylating agents induces DNA damage. It is well documented that ARTD1, ARTD2 and to a lower extent also ARTD3 recognize and bind to single- and double-strand breaks but also abnormal DNA structures, thereby get activated and start forming PAR ${ }^{2-}$ 7. PARylation marks the sites of DNA damage and facilitates DNA repair to restore cellular homeostasis ${ }^{8}$. While adequate DNA repair promotes cell survival and lowers the risk of oncogenic transformations, inhibition of PARylation by PARP inhibitors, knock-out or knock-down of ARTD1 sensitizes cells to DNA-damaging agents ${ }^{8-10}$.

Increasing genotoxic stress levels correlate with increasing ARTD1 activity, ultimately resulting in ARTD1 hyper-activation (Box 1 and 2). The fact that ARTD1 was among the first identified caspase "death substrates", suggested a regulatory role during apoptosis ${ }^{11}$. However, pharmacologic inhibition or generation of a knock-in mouse expressing a non-cleavable ARTD1 neither influenced cellular susceptibility nor the execution of the apoptotic program itself, indicating that caspase-mediated cleavage of ARTD1 and subsequent inhibition of its enzymatic activity has another physiological role ${ }^{12,13}$. DNA fragmentation is a hallmark of apoptosis, which would lead to ARTD1 hyper-activation and energy expenditure. Thus, ARTD1 cleavage during apoptosis precludes its activation and preserves energy for ATP sensitive steps during apoptosis ${ }^{12,14}$.

In contrast to ARTD1's passive role during apoptosis, PARylation actively influences cell fate during necrosis. Extensive PARylation by hyper-activated ARTD1 decreases cellular NAD levels by up to $20 \%$ within 5-15 mins and is considered the main $\mathrm{NAD}^{+}$catabolizing process in the cell ${ }^{15-17}$ (Box 
2). To balance decreased $\mathrm{NAD}^{+}$levels the cells activate adenosine triphosphate (ATP)-dependent salvage pathways resulting in ATP depletion and an comprised metabolic state of the cell ${ }^{18}$. Emerging concepts describe ARTD1 as a sensor for cellular stress levels and PARylation as a proxy signal that critically determinates cell fate ${ }^{12,19,20}$. Yet, other studies challenge the $\mathrm{NAD}^{+}$exhaustion model as the only reason for cell death. Although after middle cerebral artery occlusion (MCAO)-induced ischemia ARTD1 knock-out mice have reduced infarct sizes compared to wild-type animals, the energy status of the cells were similar ${ }^{21}$. Moreover, when UV treated, PARG-deficient cells underwent cell death without $\mathrm{NAD}^{+}$exhaustion ${ }^{22}$. Thus, PARylation-induced $\mathrm{NAD}^{+}$consumption is not exclusively responsible for cell death ${ }^{23}$. The initial observation that ARTD1 hyper-activation resulted in cell death by the release of the apoptosis-inducing factor (AIF) from the mitochondria, led to the definition of parthanatos as a new necroptosis module ${ }^{24}$. Experiments in neurons and HeLa cells subjected to genotoxic stress or the ectopic delivery of in vitro generated PAR to cells identified the PAR polymer as major contributor to AIF release and subsequent cell death ${ }^{25-27}$. Characteristically, cells dying from Parthanatos, like other necrotic cells lose their membrane integrity and thereby release intracellular material including DAMPs into the extracellular space ${ }^{1}$.

The high mobility group box 1 protein (HMGB1), a DNA binding protein that stabilizes nucleosomes and regulates transcription ${ }^{28}$, is one of the most studied DAMPs and is recognized by tolllike receptor 4 (TLR4) and the receptor for advanced glycation endproducts (RAGE). In necrosis and autophagy, HMGB1 is released in a PARylation dependent manner ${ }^{29,30}$. Mechanistically, a recent publication identified that ARTD1 is activated in macrophages by ROS and/or MAPK-signaling and PARylates HMGB1 to facilitate its acetylation-dependent release ${ }^{31}$. Hence, ARTD1 does not only regulate cell fate, but also triggers immune responses via HMGB1.

\section{Involvement of ADP-ribosylation during the immune response initiation}

Not only immune cells such as the antigen presenting macrophages or dendritic cells but also epithelial, endothelial and fibroblast cells express pathogen recognition receptors (PRRs) whose stimulation leads to initiation of innate immune responses ${ }^{32}$. PRRs recognize a variety, yet a defined set of conserved pathogen-associated molecular patterns (PAMPs), such as bacterial and fungal cell wall components (i.e. lipopolysaccharide, LPS) or DAMPs (Box 3). The recognition of DAMPS and PAMPs, often by the same receptors, activates intracellular signaling pathways, which are controlled by different ARTDfamily members and results in the expression of pro-inflammatory cytokines such as IL-12, IL-23, IL-6 and IL- $1 \beta^{32}$. The following chapter focuses mainly on reports that involved inflammatory stimuli and cells, mostly excluding findings from tumor research. For information about tumor cell signaling, readers are advised to consult respective reviews ${ }^{19,20,33}$.

ARTD1 co-activates $N F-\kappa B$-dependent gene expression:

While both the recognition of PAMPS (i.e. LPS) or DAMPs (i.e. HMGB1) by TLRs and also the TLRinduced cytoplasmic signaling events in different cell types do not seem to be regulated by ARTs, several reports have described the involvement of ARTD1 during the transcriptional activation of proinflammatory genes by PAMPs. In LPS- but also TNF- $\alpha$-stimulated macrophages ${ }^{34-37}$, microglia ${ }^{38,39}$, fibroblasts $^{35,38,40,41}$, endothelial cells ${ }^{39}$ and smooth muscle cells $\mathrm{s}^{34,36,41}$, ARTD1 was found to promote 
the NF-kB-dependent expression of pro-inflammatory cytokines. NF- $\kappa B$ is the most studied inflammatory transcription factor so far ${ }^{42}$. Different mechanistic models have been reported to explain how ARTD1 influences NF-kB-dependent gene expression. ARTD1 acts as a transcriptional co-factor for NF-KB and promotes gene expression by functional cooperation with the transcription machinery in response to pro-inflammatory stimuli ${ }^{43}$. LPS- and TNF- $\alpha$-induced NF-KB-dependent gene expression in macrophages and fibroblasts was enhanced by ARTD1 independently of its enzymatic activity, but by promoting complex formation between the mediator complex, p300/CBP and the p50 and p65 subunit of NF-kB ${ }^{44,45}$. Mutating the ARTD1 cleavage site Asp214 rendered ARTD1 uncleavable, inhibited its release from chromatin and prevented chromatin decondensation, thereby restraining the expression of cleavage-dependent NF- $\mathrm{kB}$ target genes. Interestingly, knock-in mice expressing the non-cleavable ARTD1 (D214N) mutant were highly resistant to endotoxic shock and to intestinal and renal ischemia-reperfusion. This was associated with reduced inflammatory responses in the target tissues and cells due to the compromised production of specific inflammatory mediators (e.g TNF, GM-CSF, IL-6 or LIF) ${ }^{13,46}$. These findings propose an apoptosis-independent regulatory role for caspase 7-mediated ARTD1 cleavage in pro-inflammatory gene expression. In contrast to these reports suggesting that only the protein itself but not the enzymatic activity of ARTD1 is required for NF- $\mathrm{KB}$ target gene expression, another group reported that LPS treatment of macrophages induced ARTD1's enzymatic activity and nucleosome remodeling at promoters of pro-inflammatory genes, directly destabilizing histone-DNA interactions and facilitating NF- $\mathrm{KB}$ binding and gene expression ${ }^{47}$. The discrepancies could be explained by methodological differences such as the cell type (RAW.267.4 macrophages and primary BMDM or fibroblasts), the source of LPS (S. enterica and E. coli) or the serum starvation overnight prior to LPS stimulation.

The mRNA of pro-inflammatory mediators such as $I l 1 b^{48}, I l 6^{49}, T n f a^{38,39}$ and $C x c l 2^{41}$ and adhesion molecules such as Icam1, Vcam or Eselectin ${ }^{49,50}$ were significantly lower expressed in ARTD1 knock-out cells. In LPS-stimulated glial cells, ARTD1 also enhances the DNA-binding of the transcription factors SP-1, YY-1, AP-1 and STAT1 independently of its enzymatic activity ${ }^{50}$. Although the exact mechanisms are unknown, the mRNA expression of $I l 6, I l l b$ and $T n f a$ was markedly reduced in ARTD1 knock-out glial cells ${ }^{50}$. These findings are supported by the fact that ARTD1 knock-out mice are resistant to LPS-induced shock ${ }^{40}$. So far, other ARTD family members have not been implicated in the initiation of inflammation. The fact that ARTD1 knock-out mice are resistant to endotoxic shock suggests a dominant role of ARTD1 during inflammatory signaling.

ADP-ribosylation regulates $m R N A$ stability:

Posttranscriptional regulation, particularly via mRNA stability is important for immune effector proteins to either re-program gene expression at a global level or modulate the stability and translation of specific immune transcripts ${ }^{51}$. Indeed, ARTD1 was identified to promote mRNA stability of individual transcripts during LPS-induced pro-inflammatory signaling in an enzymatically dependent manner $^{52}$. Upon LPS stimulation of macrophages, adenylate-uridylate-rich element-binding protein embryonic lethal abnormal vision-like 1 (Elavl1)/human antigen $\mathrm{R}$ (HuR) was described to be PARylated ${ }^{53}$. ARTD1-mediated PARylation of HuR enhanced nucleoplasmic shuttling and mRNA 
binding, and promoted mRNA stability ${ }^{54}$. Comparably, pharmacologic inhibition of ARTD1 reduced mRNA stability of e.g. Cxcl2 during inflammation ${ }^{52}$.

Additionally, LPS-induced expression of tissue factor (TF) is an important link between inflammation and thrombosis. In LPS-treated macrophages, ARTD8 was identified to negatively regulate $T f$ mRNA stability, by forming a complex with the mRNA destabilizing protein tristetraprolin (TTP) and a conserved adenylate-uridylate-rich element in the $T f$ mRNA $3^{\prime}$ untranslated region resulting in $T f$ mRNA degradation ${ }^{52}$. Besides ARTD8, no other ARTD family member was described to affect mRNA stability so far.

\section{Regulation of the modulation phase of the immune response by ADP-ribosylation}

The cytokines and chemokines that are expressed during the initiation of an immune response activate and recruit lymphoid cells, including innate lymphoid cells (ILCs) such as Natural Killer cells (NK cells) but also adaptive immune cells like $\mathrm{T}$ and $\mathrm{B}$ cells (Box 3 ). For example, during the amplification and modulation phase of an immune response, NK cells produce high levels of IFN- $\gamma$ to amplify the immune response and promote Th1 immune polarization. Additionally, IL-12 and IFN- $\gamma$ promote the differentiation of naïve CD4 T cells into T helper type 1 (Th1) cells expressing additional IFN- $\gamma$, and TGF $\beta$ promotes differentiation into regulatory $\mathrm{T}$ cells (Treg), while TGF $\beta$, IL-6, IL-1 $\beta$ and IL-23 together induce $\mathrm{T}$ helper type 17 (Th17) cells.

\section{ADP-ribosylation controls $T$ cell activation and survival:}

Bone marrow-derived lymphoid progenitor cells migrate to the thymus to give rise to $\mathrm{T}$ cells, which will subsequently migrate to peripheral lymphoid tissues where a critical balance between cell division and cell death must be maintained to ensure $\mathrm{T}$ cell homeostasis throughout life ${ }^{55}$. With respect to $\mathrm{T}$ cell development, $\mathrm{Artd}^{-/}{ }^{-}$mice were reported to have a two-fold lower number of CD4/CD8 double positive (DP) thymocytes due to decreased cell survival and increased apoptosis ${ }^{56}$. Although ARTD1 and ARTD2 do not influence peripheral $\mathrm{T}$ cell homeostasis individually, the deficiency of both enzymes lead to severe DNA damage, cell death and diminished T cell numbers in peripheral lymphoid tissues $^{57}$. In addition, T-dependent antigen-treated (TNP-KLH) Cd4-Cre;Artd $2^{\text {floxflox }} ;$ Artd $^{-/}$mice exhibited defective antibody responses as determined by decreased IgM and IgG1 serum levels 14 days after treatment, suggesting defective $\mathrm{T}$ and $\mathrm{B}$ cell cooperation ${ }^{57}$.

Membrane receptors are crucial initiators of signal transduction in $\mathrm{T}$ cells and activate $\mathrm{T}$ cells to proliferate and differentiate ${ }^{58}$. Necrotic cells that have lost their membrane integrity release $\mathrm{NAD}^{+}$to the extracellular space. In presence of extracellular $\mathrm{NAD}^{+}$, ectopically expressed ARTC2.2 mediates ADP-ribosylation of the purinergic $\mathrm{P} 2 \mathrm{X} 7$ receptor at Arg125 on CD4 and CD8 T cells in mice, which induces a $\mathrm{Ca}^{2+}$ influx, macropore formation, phosphatidyl-serine exposure, CD62L shedding, and accelerated cell death ${ }^{59,60}$. ARTC2.2 was also reported to modify Arg35 on the high affinity receptor for IL-2, CD25, leading to a down-modulation of IL-2 signaling which prevents development and activation of regulatory $\mathrm{T}$ cells ${ }^{61}$. Additionally, necrosis-activated ARTC2.2 modifies membrane proteins including LFA-1, CD8, CD27, CD43, CD44 and CD45 on the surface of CD8 cytotoxic T cells, resulting in decreased proliferation and decreased cytotoxicity ${ }^{62}$. Thus, ARTC2.2 acts as a safeguard in mice to prevent undesirable $\mathrm{T}$ cell activation during necrosis. 
ADP-ribosylation regulates gene expression in activated $T$ cells:

TGF $\beta$, IL-6, IL-1 $\beta$ and IL-23 promote naïve CD4 T cell differentiation towards pro-inflammatory Th17 cells that are characterized by the expression of IL-17 and IL-22 ${ }^{63}$. In contrast, TGF- $\beta$ and IL-2 drive naïve $\mathrm{CD} 4 \mathrm{~T}$ cells differentiation into $\mathrm{CD} 4{ }^{+} \mathrm{CD} 25^{+}$regulatory $\mathrm{T}$ cells (Treg) that play an important role for immune homeostasis ${ }^{64,65}$. In CD4 T cells ARTD1 represses the expression of both subunits of the TGF- $\beta$ receptor: whereas the expression of TGFbRI is repressed by ADP-ribosylation, the expression of TGFbRII is repressed by the ARTD1 protein independently of its enzymatic activity $^{66}$. ARTD1-deficient CD4 T cells expressed increased levels of both TGF- $\beta$ receptors, which was associated with enhanced Smad2/3 activation and up-regulation of FoxP3, the master transcription factor of $\mathrm{T}$ helper cell differentiation ${ }^{66}$. Further studies confirmed an inhibitory role for ARTD1 during Treg differentiation, as shown by increased numbers of FoxP3 ${ }^{+}$Tregs in lymphoid organs of ARTD1 knock-out mice compared to wild-type mice ${ }^{67,68}$.

$\mathrm{T}$ cell activation is not only driven by cytokines but also by the binding of the $\mathrm{T}$ cell receptor (TCR) to major histocompatibility complex (MHC)-bound antigens on cells. TCR activation triggers the activation of receptor-associated tyrosine kinases that lead to the activation of phospholipase $\mathrm{C}-\gamma$ (PLC- $\gamma)$ signaling cascade, intracellular $\mathrm{Ca}^{2+}$ release and activation of nuclear factor of activated $\mathrm{T}$ cells (NFAT) transcription factors ${ }^{69}$. During PMA/ionomycin-induced $\mathrm{T}$ cell activation (which mimics the TCR activation), ARTD1 was shown to interact with and modify NFATc1 and NFATc2 in the nucleus $^{70}$. In particular, for NFATc2 the PARylation site could be determined at its regulatory region spanning amino acids $1-460^{70}$. Nuclear NFAT PARylation delays its nuclear export and thus enhances NFAT-dependent gene expression. As a consequence, ARTD1 knock-out T cells exhibit markedly reduced $I l 2$ and $I l 4$ expression $^{71}$.

In addition, anti-CD3 or anti-CD3/anti-CD28 mediated T cell activation implicate ARTD1 in $\mathrm{T}$ cell polarization. Microarray-mediated transcriptional profiling revealed increased Th1 cytokine (Ifng) and chemokine ( $\mathrm{Cxcll}, \mathrm{Ccl} 4$ and Ccl9) mRNA expression and reduced Il4 mRNA expression in ARTD1 knock-out T cells ${ }^{72}$. However, it was not elucidated in this study whether ADP-ribosylation or the pure presence of the ARTD1 protein is required for the posttranscriptional control during the amplification and modulation phase of the immune response. Although other studies provide evidence that inhibition of ADP-ribosylation by PJ34 inhibits anti-CD3/anti-CD28-stimulated Il2 and INF expression in $\mathrm{T}$ cells, this effect seemed to be independent of ARTD1 or ARTD2 ${ }^{73}$. Furthermore, also for the NK cell function during the amplification phase it is not known whether ADP-ribosylation is implicated in the regulation.

\section{Participation of ADP-ribosylation during the effector phase of the immune response}

Severe pathogenic infections usually cause tissue damage, the release of DAMPs and secondary inflammatory reactions ${ }^{74}$. To defeat infections, macrophages produce high levels of nitric oxide (NO) that lyses the pathogen, neutrophils are recruited to the site of infection to produce antimicrobial oxygen radicals and epithelial/endothelial cells close their tight junctions to prevent the spreading of the pathogen ${ }^{74}$. In contrast to these innate responses, cells of the adaptive immune system, such as cytotoxic CD8 $\mathrm{T}$ cells specifically recognize infected cells by TCR-MHC-I interactions ${ }^{75}$. Additionally, CD4 $\mathrm{T}$ cells modulate the adaptive immune responses, both cytotoxic and antibody 
responses, by secretion of a specific set of cytokines. The second main arm of the adaptive immune system consists in antibody-producing B cells that can specifically clear invading pathogens and can also establish immunological memory.

ADP-ribosylation regulates effector cell-specific gene expression and function:

In macrophages, ARTD1 functions as co-factor of NF-kB (see above) to enhance inducible nitric oxide synthase (iNOS) expression ${ }^{35}$. Beside its cytotoxic role during pathogen killing, increased NO production can also lead to increased genotoxic stress for non-infected neighboring cells. Thus, during severe pathogen infections ARTD1 is believed to increase the genotoxic stress of these cells via iNOSmediated NO production, ultimately driving them into necrosis ${ }^{76}$, thereby linking bacterial infections and parthanatos. Thus, ARTD1 drives tissue injury in a positive feedback loop and serves as a critical immune checkpoint for the host to prioritize pathogen elimination over cell death ${ }^{77}$. A recent publication identified ARTD8 and ARTD9/BAL1/PARP9 as opposing regulators during macrophage polarization ${ }^{78}$. Whereas ARTD8 depletion during $\mathrm{M}(\mathrm{IFN}-\gamma)$ polarization enhanced STAT1 phosphorylation and gene expression of inflammatory cytokines (i.e. TNF- $\alpha$ and IL-1 $\beta$ ), ARTD9 depletion resulted in decreased STAT1 phosphorylation and decreased pro-inflammatory gene expression $^{78}$. Mechanistically, the authors provided evidence that ARTD8 ADP-ribosylated STAT1 at the C-terminal amino acid (AA) residues Glu657 and Glu705, which was abolished in the presence of ARTD9. The expression of ADP-ribosylation deficient mutants of STAT1 enhanced its phosphorylation and the expression of Nos2, Illb and $C c l 2^{78}$. Additionally, during M(IL-4) polarization, ARTD8 depletion reduced STAT6 phosphorylation and Arg1 expression ${ }^{78}$. Thus, ARTD8 suppresses $\mathrm{M}(\mathrm{IFN}-\gamma)$ polarization and enhances M(IL-4) polarization.

During IL-1 $\beta$ and TNF- $\alpha$ mediated signal transduction, ARTD10 was identified to play an important role in NF- $\mathrm{KB}$ activation ${ }^{79}$. Both cytokines initiate the tumor-necrosis factor receptorassociated factor (TRAF) protein, which function as K63-specific E3 ubiquitin ligase synthetizing K63-pUB chains on TRAF itself. ARTD10 binds to K63-pUb, MARylates NF- $\mathrm{kB}$ essential modulator (NEMO) whereby its polyubiquitination and subsequent degradation is inhibited. The degradation of NEMO would release IKK- $\alpha$ and IKK- $\beta$ to phosphorylate IKB allowing nuclear translocation of NF-

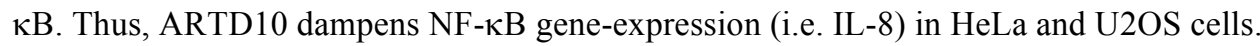

Neutrophils produce antimicrobial human neutrophil peptides (HNPs) to eliminate pathogens ${ }^{80}$. ARTC1 expression is markedly increased in chemotactically stimulated neutrophils (i.e. by fMLP, IL-8 or the platelet activating factor) ${ }^{81}$, which has a profound impact on neutrophil effector function ${ }^{82}$. Indeed, ARTC1 modifies HNP-1 leading to inhibited antimicrobial activity in vitro, while retaining its $\mathrm{T}$ cell chemoattractant abilities ${ }^{83,84}$.

Microglia exhibit similar properties, such as Fc-receptor-mediated phagocytosis, like peripheral macrophages but reside locally in the central nervous system (CNS). With a mass spectrometry approach a recent publication identified ARTC2.1 to be upregulated in LPS and U0126 (ERK1/2 inhibitor) stimulated microglial cells in vitro ${ }^{85}$. In addition, various cell surface proteins were identified to be MARylated and the modification of Fc-receptors (Fc $\gamma R$ 1 and Fc $\gamma R 2 B$ ) significantly inhibited IgG binding and the phagocytosis of IgG-coated microbeads. The data suggests that ectopic 
ADP-ribosylation may regulate myeloid cell function ${ }^{85}$. It will be interesting to investigate the functional role of other ARTC targets during the effector phase of myeloid cells in vivo.

ADP-ribosylation influences immunoglobulin class switching in B cells:

Antibodies are produced by B cells and are central mediators of the adaptive immunity, since they neutralize pathogens and pathogen-derived products, and they recruit cellular immune effectors to eliminate infections ${ }^{86}$.

Under basal conditions, ARTD1 inhibition or ARTD1 knock-out increases class switching frequency to $\operatorname{IgA}$ and $\operatorname{IgG} 2 \mathrm{~b}$ and decreases the switching frequency to $\operatorname{IgG} 2 \mathrm{a}$ by unknown mechanisms ${ }^{87}$. Although this study did not analyze whether ARTD1's catalytic activity is required for the observed phenotype, the authors speculated about ARTD1's function as a co-factor during the DNA double-strand forming class switching process ${ }^{87}$. Immunoglobulin class switching is also induced by cytokines. In IL-4-stimulated B cells, ARTD8 was identified as a transcriptional switch during STAT6-dependend gene activation ${ }^{88}$. Mechanistically, in the absence of the class-switch stimulating cytokine IL-4, ARTD8 associates with histone deacetylase (HDAC) 2 and HDAC3 to repress $I \varepsilon$ (IgE) gene expression. IL-4 activates ARTD8 and leads to the dissociation of the HDACs from the promoter and to the expression of $I \varepsilon$, as well as to an $\operatorname{IgM}$ to $\operatorname{IgE}$ class switching ${ }^{88}$.

\section{The role of ADP-ribosylation erasers during inflammation}

Erasers degrade ADP-ribosylation by cleaving glycosidic bonds to generate mono-ADP-ribose that eventually serves as a substrate for ATP production ${ }^{89}$. Hence, erasers are the biochemical and physiological counterpart of writers (i.e. ARTs) and are involved in the ADP-ribosylation metabolism. The eraser family includes ARH1, ARH2, ARH3, MacroD1, MacroD2, TARG and the founding member PARG ${ }^{42}$.

The evidence of erasers in modulating the immune response is so far marginal. Only PARG has been implicated in inflammatory diseases and will thus be discussed here (Box 1). Strikingly, both inhibition and genetic ablation of ADP-ribosylation writers (i.e. ARTD1) and erasers (i.e. PARG) protect against many inflammatory diseases. So far, PARG has been implicated in different PAMP- and DAMPassociated inflammation models, such as peritonitis, LPS-induced septic shock, dinitrobenzene sulfonic acid (DNBS)-induced colitis and oxidative-stress induced neuronal cell death ${ }^{90}$, however, without providing underlying molecular mechanisms. During pathogenic infections PARG has opposing functions: while PARG inhibition by GPI 18214 protects mice during zymosan (yeast cell surface glucan)-induced peritonitis by decreased neutrophil infiltration into the lungs and reduced plasma TNF$\alpha$ and IL-1 $\beta$ levels ${ }^{91}$, PARG $_{110^{-/}}$are hypersensitive to LPS-induced septic shock potentially due to the still expressed enzymatically inactive $\mathrm{PARG}_{60}$ isoform ${ }^{92}$.

PARG has also been suggested to promote ischemia-reperfusion-induced tissue injuries. For example, PARG inhibition or genetic ablation of PARG in mice and rats decreased neutrophil infiltration, endothelial expression of ICAM-1, P-Selectin and TNF- $\alpha$ plasma levels during gastrointestinal ischemia ${ }^{93}$. Moreover, PARG inhibition decreased the infarct size in a rat-model of cerebral ischemia reperfusion ${ }^{94}$. In a model of renal ischemia reperfusion, $\mathrm{PARG}_{110^{-/}}$mice exhibited 
significantly reduced tissue injury and dysfunction ${ }^{95}$. Analogously to ARTD1, PARG does also promote IBD. $\mathrm{PARG}_{110^{-/-}}$mice were resistant and PARG inhibitor treated mice were significantly protected in a model of DNBS-induced colon injury ${ }^{96}$. Mutant mice exhibited significantly lower colon TNF- $\alpha$ and IL-1 $\beta$ levels. Overall, currently available data suggests important functions of PARG during distinct inflammatory conditions that are worthwhile pursuing in the future. Especially PARG inhibitors could open new therapeutic opportunities for treating inflammatory diseases. However, functional analyses to understand the molecular mechanisms of the above-mentioned phenotypes are critically needed. Moreover, the role of other erasers e.g. ARH3 or MDO1/2 during inflammation might be an attractive target for further investigations.

\section{ADP-ribosylation as anti-viral defense mechanism}

Viruses only replicate in infected host cells, hence "hijack" the cellular machinery to generate new viral particles. They induce an immune response that is determined by the balance between two competing processes: (i) the ability of cells to sense virus-specific PAMPs and (ii) the ability of viral proteins to interfere with the cellular response to viral infection ${ }^{97}$. In fact, cells rely on ARTD family member-dependent defense mechanisms during different phases of viral replication. Interferonstimulated cells are primed for antiviral responses and express various antiviral genes. Recently, Zhang et al identified the ARTD9-deltex E3 ubiquitin ligase 3L (DTX3L) interaction as a potent antiviral mediator $^{98}$. During interferon signaling, ARTD9-DTX3L and STAT1 interaction activates the E3 ubiquitin ligase to modify histone $\mathrm{H} 2 \mathrm{~B}$ at distinct chromatin loci and facilitate host pro-inflammatory gene expression. In addition, ARTD9-DTX3L E3 ubiquitin ligase activity also targeted the viral 3C protease degradation by the immunoproteasome. Thus, ARTD9-DTX3L targets host and viral proteins to promote viral clearance.

After successful entry into a host cells, viral mRNA needs to be expressed by the host transcriptional machinery. In an elegant search for novel host antiviral factors, Gao et al. identified a Zinc-finger Antiviral Protein, today known as the catalytically inactive ARTD13, as a viral RNA binding protein ${ }^{99}$. Interaction studies identified two RNA helicases (p72 and DHX30) and exosome components to associate with ARTD13, suggesting that ARTD13 regulates viral RNA unwinding and exosome degradation ${ }^{100}$. ARTD13 is now known to inhibit various retroviruses, including human immunodeficiency virus 1 (HIV-1), or other RNA viruses such as Alphaviruses and Filoviruses ${ }^{100}$. Host antiviral protein expression during infection is crucial for viral elimination. In plants and invertebrates, the expression of these transcripts is in part regulated post-transcriptionally by $\mathrm{RNAi}^{101}$. In eukaryotic virus infected cells, ARTD13/ZC3HAV1/PARP13 was reported to reduce RNAi activity and promoted the expression of interferon-stimulated genes (ISGs). Mechanistically, the authors observed a correlation between the poly-ADP-ribosylation of the RNA-induced silencing complex (RISC) and ARTD $13^{101}$. Since ARTD13 is enzymatically inactive ${ }^{102}$, we propose that another ARTD family member PARylates RISC in an ARTD13 dependent manner.

Recently, ARTD14/PARP7 has been identified to sense viral RNA as well as to initiate viral RNA degradation in an exosome-dependent manner ${ }^{103}$. Thereby, ARTD14 inhibits Sindbis Virus replication in host cells. Host ribosomes translate viral transcripts and mediate viral amplification. A recent study identified ARTD12/PARP12 to interfere with viral translation ${ }^{97}$. Mechanistically, the data 
suggests that the longer isoform of the two ARTD12 isoforms, ARTD12L as well as its catalytic activity are required for complex formation with ribosomal proteins at polysomes to efficiently inhibit translation of the venezuelan equine encephalitis virus (VEEV) $)^{97}$. In addition, a very recent publication could show that ARTD12 also interferes with Zika virus infection ${ }^{104}$. Mechanistically, ARTD12 ADPribosylates the virus nonstructural proteins NS1 and NS3 to promote their ubiquitination-dependent proteasomal degradation and inhibits viral replication.

Viral replication often occurs in the nucleus of the infected cell. Under basal conditions, ARTD5/tankyrase 1 and ARTD6/tankyrase 2 regulate telomere maintenance, WNT-signaling and mitosis ${ }^{105}$. A recent study identified that during herpes simplex virus (HSV) infection, particularly ARTD5 is phosphorylated and localized to the nuclear viral replication compartment ${ }^{106}$. HSV did not replicate in ARTD5/6-depleted cells or pharmacologically inhibited (XAV939) cells, suggesting that ARTD5/6 enzymatic activity promotes HSV replication.

\section{PARP inhibitors as anti-inflammatory compounds}

Besides having beneficial effects in oncology (Box 2), PARPi have also been implicated and tested in numerous inflammatory disease models such as neurological diseases, sepsis, chronic inflammatory diseases and reperfusion injuries (see Table 1). The inhibitors affected several molecular mechanisms described above and thereby led to reduction of cell death-mediated induction of sterile inflammation and reduced DAMP release, thus lowering tissue damage and controling pro-inflammatory cytokine expression, which plays crucial roles in immune response propagation and immune cell recruitment (Box 3).

\section{Concluding Remarks, outstanding challenges and limitations}

Many research groups with diverse expertise have embarked on ADP-ribosylation research in recent years. Despite their efforts, some very basic questions remain unanswered (Outstanding Question Box). A highest priority is (i) to elucidate the biological functions of the recently identified ARTs and ADPribosylhydrolases - cell type-specific knockout mice (i.e. conditional KOs) will help to gain a clear mechanistic understanding of which ART contributes in which cell type to what sort of processes; (ii) the other high priority topic consists in understanding PARPi action not only in tumor therapy but also in inflammatory disorders. The importance of ADP-ribosylation inhibitors is underscored by the increasing use of these compounds in clinical medicine. Although these inhibitors have been used to treat tumors based on the DNA repair activity of ARTD1, they could also be used for the treatment of inflammation-associated diseases which are in part regulated by other ARTD family members. None of the currently available inhibitors specifically inhibits only a single ARTD family member, although some progress toward this goal has been made. However, global inhibition of ARTDs may be more beneficial under certain medical circumstances, as there would no longer be any functional compensation by other ARTD family members.

The current limitations consist in (i) lack of information on the role of individual ADPribosylation sites - while for a handful of sites there is mechanistic evidence for an individual role of ADP-ribosylation sites in regulating particular processes, there is a vast and growing number of sites for which it is unknown whether their ADP-ribosylation has a mechanistic impact or is a pure epi- 
phenomeneon; (ii) the distinction of MAR and PAR structures at specific sites is still not possible thus, it is unknown whether mono-, oligo- or linear and branched poly-ADP-ribosylation of a specific site can exist and have differential outcomes; (iii) generally, tools to study ADP-ribosylation by nonexpert labs are missing, which is a main reason why mechanistic information on ADP-ribosylation is scarce. Since there is still a huge gap between the mechanistic understanding of ADP-ribosylation events and interference with ADP-ribosylation in diseases by e.g. PARP inhibitors, a gap that constitutes an obstacle for tackling clinical trials in inflammatory disorders.

We have only just begun to understand the biochemistry of ADP-ribosylation reactions during inflammation. Increased basic and preclinical research is needed to advance the use of PARPi in the treatment of tumors and potentially in inflammation-associated diseases. Further development of tools and methods will greatly contribute to our future understanding of the complex functions of ADPribosylation and how it regulates physiological and pathological processes. 


\section{Glossary}

AIF

AIF is a high affinity PAR binding protein which localizes to the mitochondrial intermembrane space where it plays essential roles in the mitochondrial respiratory chain. A smaller pool of AIF associates to the outer mitochondrial membrane (cytosolic side). Its release is a critical step for different modes of programmed cell death.

\section{ARTs}

ADP-ribosyltransferases are a class of enzymes that occur in bacteria, viruses and all mammalian cells except for yeast. They catalyze the post-translational modification ADP-ribosylation.

Chromatin

Chromatin describes the association of nucleic acids (DNA/RNA) and proteins (mostly histones) to organize the genetic information.

\section{DAMPs}

Danger associated molecular patterns are structural components of the host (e.g. the nuclear protein HMGB1) that are released from necrotic cells and initiate a response to clear the cellular debris and to remodel the affected tissue.

\section{Death substrates}

Programmed cell death such as apoptosis is executed by caspases (cysteine-aspartic proteases). In a particular order of events, the enzymes cleave distinct substrates, so called death substrates.

\section{Genotoxic stress}

Cells are constantly exposed to genotoxic stressors such as UV-light, radiation or chemical agents, which induce mutations in the DNA. The DNA repair machinery resolves these lesions that eventually could give rise to transformed cells and cancer.

\section{Macrophage polarization}

Macrophages were found to exhibit a wide range of effector functions depending on the polarization by different cytokines. The most prominent macrophage types are pro-inflammatory M1-like (IFN $\gamma$ ) and anti-inflammatory/regulatory M2-like (IL4) ${ }^{107,108}$.

\section{NAD}

Nicotinamide dinucleotide is a coenzyme which carries 2 electrons in its reduced form of NADH. It plays an important role in der cellular energy metabolism and serves as the substrate for ADPribosylation.

\section{$\mathrm{NF}-\kappa \mathrm{B}$}

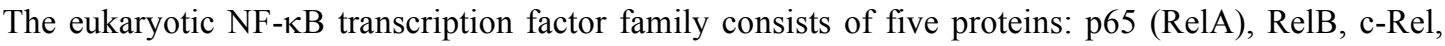
p105/p50 (NF-kB) and p100/52 (NF-KB1). Dimerization of the different subunits can result in 15 different combinations, while the p50/p65 heterodimer is the most abundant one. In its inactive form the p50/p65 heterodimer is bound by IKB, inhibiting its nuclear translocation. TLR-triggering induces

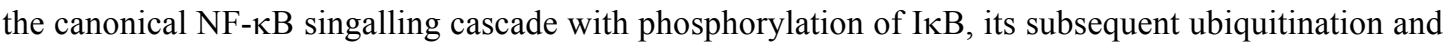
degradation. In turn, the released p50/p65 translocates into the nucleus and initiates gene expression. 
Pathogen associated molecular patterns are structural components of foreign bacteria (e.g. LPS), viruses or parasites that are recognized by conserved host receptors to identify foreign elements within the body and to license the initiation of an immune response to clear the infection.

\section{Parthanatos}

This caspase-independent mode of cell death combines apoptotic and necrotic features. The name derives from par for PAR polymer, and Thanatos the personification of death in Greek mythology. It is induced by the release of complex PAR chains from the nucleus resulting in mitochondrial depolarization and AIF release.

\section{PARylation/MARylation}

Some ARTs attach a single ADP-ribose moiety to target proteins, which is called mono-ADPribosylation or MARylation. Other ARTs can extend the initial modification to poly-ADP-ribosylation, or PARylation, which can be linear or branched.

\section{Synthetical lethality}

The concept describes a genetic interaction in which single-gene defects are compatible with cell viability, but the combination (or synthesis) of gene effects results in cell death. BRCA mutated cells are up to 1000 times more sensitive to PARPi than wild-type cells

\section{ACKNOWLEDGMENTS}

We would like to thank Zuzanna Kotkoswka and in particular Tobias Suter (University of Zurich) for the helpful discussions, suggestions and for providing editorial assistance also including visualization of the figures. ADP-ribosylation research in the laboratory of $\mathrm{MOH}$ is funded by the Kanton of Zurich and the Swiss National Science Foundation (grant 310030_157019).

We apologize to the colleagues whose work could not be discussed due to size limitations.

\section{AUTHOR CONTRIBUTIONS}

F.A.K. and M.O.H. wrote the manuscript and designed the figures.

\section{COMPETING FINANCIAL INTERESTS}

The authors declare no competing financial interests. 


\section{Highlights Box}

- ADP-ribosylation serves as a checkpoint control for cell fate and actively drives a type of regulated cell death (parthanatos) to induce inflammation.

- ARTD1-mediated PARylation affects mRNA stability of pro-inflammatory cytokines.

- Dual function of ARTD9-DTX3L interaction as potent antiviral mediator via chromatin modification and viral protein degradation.

- ARTD8 and ARTD9 regulate macrophage polarization via STAT1 phosphorylation and ADPribosylation.

- ARTD10-mediated MARylation of NEMO inhibits its degradation consequently dampening NF- $\mathrm{BB}$ gene expression.

- Ectopic ARTC2.1 on microglia regulates phagocytosis by modifying immunoglobulin receptors.

- ARTC2.2 modifies CD25 and P2X7 on T cells thereby leading to dampening of IL-2 signaling and to NAD-induced cell death (NICD), respectively. 


\section{Box 1. Biology of ADP-ribosylation}

ADP-ribosylation is an evolutionary conserved post translational modification (PTM) that is present in many prokaryotic and all eukaryotic cells, except for yeast ${ }^{109}$. The PTM is catalyzed by ADPribosyltransferases (ARTs) that covalently attach ADP-ribose (ADPr) to amino acid residues of target proteins using nicotinamide adenine dinucleotide $\left(\mathrm{NAD}^{+}\right)$as substrate (Figure below). Bacterial toxins such as from C. diphteriae, V. cholerae, B. pertussis or C. botulinum mono-ADP-ribosylate host proteins to inhibit their function and promote bacterial pathogenesis ${ }^{110}$. Beside prokaryotic ARTs, various eukaryotic proteins have been described to catalyze, hence "write" ADP-ribosylation". Based on structural homologies to bacterial toxins the mammalian writers have been classified into two groups: Intracellular diphtheria toxin-like ARTs (ARTDs, formerly known as PARPs ${ }^{111}$ ) and the ectopic cholera toxin-like ARTs (ARTCs ${ }^{112}$ ). Additionally, Sirtuins (specifically SIRT4 and SIRT6) are also considered to possess ADP-ribosylating activity ${ }^{109}$. While all of the known ARTs attach a single ADPr molecule to a protein, which is called mono-ADP-ribosylation or MARylation, only few ARTs can further modify the initial ADPr with additional ADPr molecules generating linear or branched chains which is called poly-ADP-ribosylation or PARylation ${ }^{111}$.

Genome wide sequence analyses identified 18 intracellular eukaryotic ARTDs that share a common "PARP signature" motif in their C-terminal ART domain ${ }^{23}$. While four members (ARTD1, 2, 5, 6) catalyze PARylation, twelve members (ARTD3, 4, 7, 8, 10, 11, 12, 14, 15, 16, 17 and 18) catalyze MARylation. For ARTD9 and ARTD13, no enzymatic activity has been described so far ${ }^{111}$. ARTDs localize to various intracellular compartments and eventually associate with distinct cellular structures $^{111}$. Whereas ARTD1 strictly localizes to the nucleus, ARTD2-6, ARTD8-11 and ARTD14 localize to nucleus and cytoplasm, ARTD7, 12, 13 and 15-17 predominantly localize to the cytoplasm $^{42}$. Apart from their characteristic catalytic domain, ARTDs are structurally very unique which suggests diverse biological functions ${ }^{51}$.

The ARTC family, also known as ecto-ARTs or GPI-linked NAD(P) ${ }^{+}$-arginine ADPribosyltransferases, consists of 4 members in human and 6 members in mouse. Members of the ARTC family contain the characteristic cholera toxin motif in their active center. ARTCs are facing the extracellular site of the cell and are bound to the plasma membrane by a glycosylphosphatidylinositol (GPI) anchor. ARTC2.1 and ARTC2.2 (also known as Art2a and Art2b respectively) are expressed only in the mouse, and are not expressed in human due to a premature stop-codon ${ }^{112}$. ARTC3 and 4 lack the R-S-E motif, which prevents them from binding $\mathrm{NAD}^{+}$and renders them catalytically inactive. ARTC5 which lacks the GPI-anchor is secreted ${ }^{83}$.

Numerous proteins possess ADPr interaction domains and are considered as "readers" of ADP-ribosylation ${ }^{109,113}$. Another set of enzymes including the PAR-degrading enzyme poly(ADPribose) glycohydrolase (PARG) hydrolase "erase" ADP-ribosylation on target proteins making ADPribosylation a fully reversible $\mathrm{PTM}^{111}$. Human PARG has five isoforms with distinct subcellular localizations: a $111 \mathrm{kDa}$ (nuclear), a $102 \mathrm{kDa}$ (cytoplasmic), a $99 \mathrm{kDa}$ (cytoplasmic), a $60 \mathrm{kDa}$ (nuclear and cytoplasmic) and a $55 \mathrm{kDa}$ (mitochondrial) isoform ${ }^{114-118}$. The murine PARG protein exists in two isoforms which differ in their N-terminal region: while wild type PARG $\left(\mathrm{PARG}_{110}\right)$ is a $110 \mathrm{kDA}$ 
protein composed of an $\mathrm{N}$-terminal regulatory domain and a C-terminal catalytic domain, the PARG mutant isoform $\left(\mathrm{PARG}_{60}\right)$ is a $60 \mathrm{kDa}$ protein that lacks the $\mathrm{N}$-terminal regulatory domain ${ }^{92,114,119}$. The ARH family consists of homologous proteins (ARH1-3) that are structurally distinct from PARG and have varying degrees of hydrolase activity and different specificities toward ADP-ribosylated targets (for review see ${ }^{120}$ ). Recently we and other provided evidence that ARH3 not only reverses PARylation, but also MARylation ${ }^{121,122}$. In addition to their ability to recognize and bind PAR and MAR attached to proteins, a subset of macrodomains also exhibit enzymatic activity and is able to demodify MARylated proteins ${ }^{123-125}$.

Protein ADP-ribosylation can either affect the activity of the modified protein or its interaction with nucleic acids and with other proteins ${ }^{33}$. Similar to other post-translational modifications such as acetylation, phosphorylation, ubiquitination, sumoylation or methylation, protein ADP-ribosylation is implicated in a plethora of different cellular processes including genomic stability, transcriptional control, energy metabolism and cell death, all of them directly affecting inflammatory conditions $^{51,126-128}$.

a)

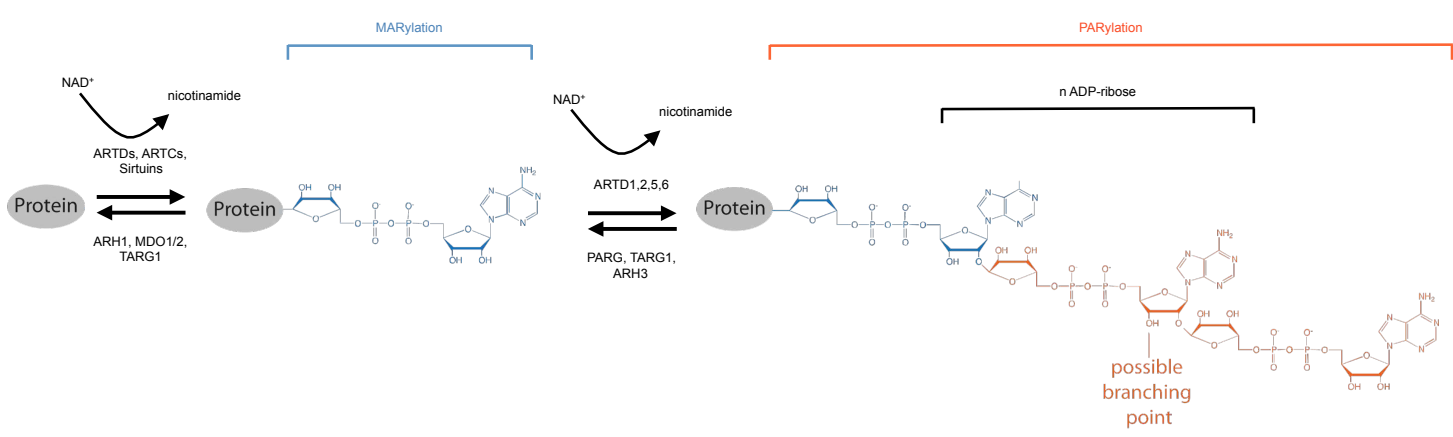

b)

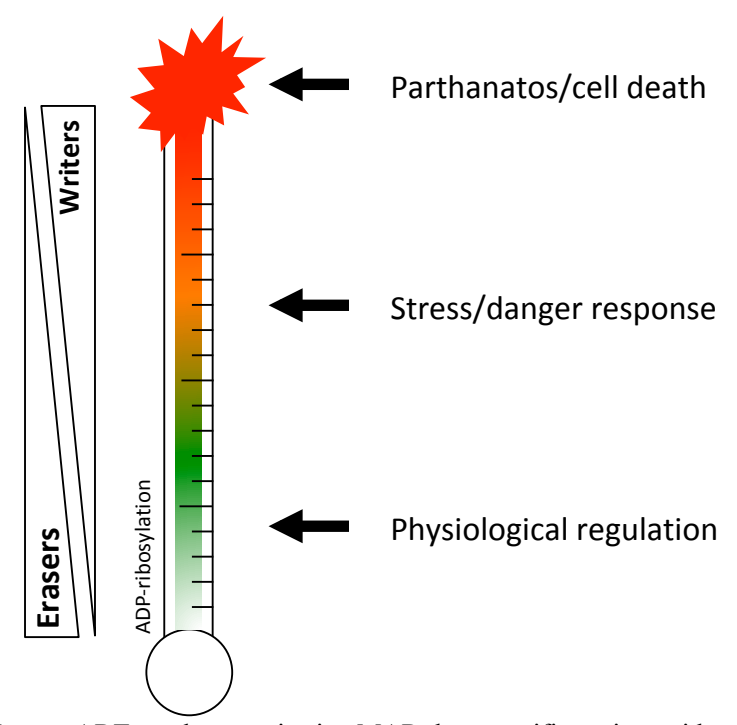

(a) The ADP-ribosylation scheme. ARTs and some sirtuins MARylate specific amino acid residues using NAD $^{+}$as substrate, which is reversed by ARH1 and MDO1/2. ARTD1, 2, 5 and 6 extend MAR to PAR, which is reversed to MAR by PARG and TARG. ARH3 is able to reverse PAR and MAR.

(b) ADP-ribosylation is a proxy signal for cell stress and determinant of cell fate. Genotoxic stress levels correlate with ARTD1 activation and ADP-ribosylation levels. Whereas basal PARylation beneficially regulates various physiological processes, extensive ADP-ribosylation not only serves as a stress/danger signal but ultimately drives cells into cell death. 


\section{Box 2. Clinical use of ADP-ribosylation inhibitors (PARP inhibitors)}

Historically, ARTD1 and ARTD2 are known for their role during DNA damage response (DDR), particularly the base excision repair (BER) pathway and double strand break (DSB) repair pathway, although both proteins likely regulate independent, but intrinsically linked aspects of DNA base damage response ${ }^{9,33,129}$. These involvements led to the development of small-molecule ADPribosylation inhibitors (PARP inhibitors; PARPi). The underlying concept was that the inhibition of the DNA-repair machinery could sensitize tumor cells to conventional DNA-damage-based therapies such as chemo- or radiotherapy ${ }^{130}$.

More than 30 years ago, nicotinamide and its derivatives, including 3-aminobenzamide (3$\mathrm{AB}$ ) were identified as the first PARP inhibitors ${ }^{130}$. Advances in drug design and screening led to the discovery of several new generations of PARPi compounds, all of them competing with $\mathrm{NAD}^{+}$. Today, some of these PARPi are clinically approved as anti-cancer agents, including veliparib (Abbvie), rucaparib (Pfizer/Clovis), olaparib (KuDOS/AstraZeneca), niraparib (Merck/Tesaro) and talazoparib (Lead/Biomarin/Medivation/Pfizer) ${ }^{76,131}$. Two cancer treatment strategies with PARPi have been suggested: either monotherapy or combination therapies of PARPi and chemo- or radiotherapy ${ }^{132}$. $B R C A$-deficient breast and ovarian cancers are particularly sensitive to PARPi. The tumor suppressors BRCA1 and BRCA2 are essential proteins for the DNA DSB repair machinery to resolve DSBs by homologous recombination (HR ${ }^{132}$. Defects in HR results in the accumulation of genetic alterations that further promotes tumorigenesis. In 2005, two independent groups discovered a synthetical lethality interaction between ARTD1, BRCA1 and BRCA2 $2^{133,134}$. Thus, BRCA mutated cells are up to 1000 times more sensitive to PARPi than wildtype cells ${ }^{134}$. Recently, another PARPi mode of action called "PARP trapping" was proposed for a subset of PARPi (especially rucaparib, olaparib, niraparib and talazoparib) ${ }^{135,136}$. Inhibitor-bound ARTD1 is "trapped" to the DNA and interferes with its function and/or cellular replication resulting in cell death ${ }^{137}$.

Although the potency and specificity of PARPi greatly improved during the last decades, ART-specific PARPi are still missing ${ }^{138}$. Interestingly, PARPi have also been reported to dampen different inflammation types, strongly corroborating the notion that ADP-ribosylation also regulates inflammation (summarized in Table 1) that might have to be taken into consideration, when treating tumor patients with PARPi ${ }^{43,130,139}$ (Figure below). At which stage of inflammation and to which extent this effect is cell type specific is currently under investigation. 


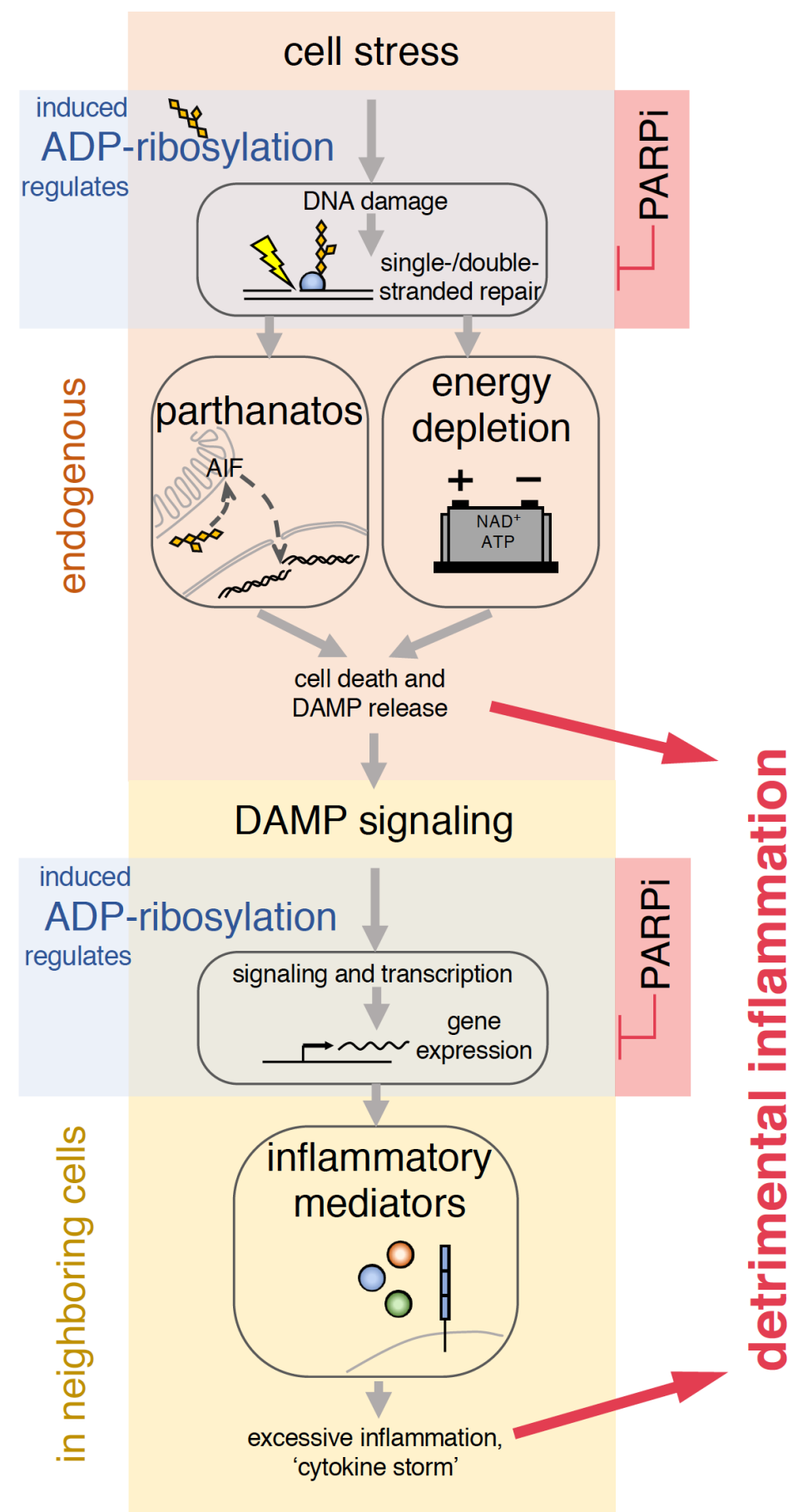

Multiple Roles of intracellular ADP-ribosylation in DNA Repair and Signaling/Transcription. ADP-ribosylation facilitates DNA single and double stand repair and its inhibition sensitizes cells to accumulate DNA damage, eventually leading to cell death. However, excessive ADP-ribosylation due to continuous cellular stress may also lead to cell death by parthanatos or energy depletion, which could imbalance the immune system. As a consequence of DAMP release, neighboring cells will also be implicated into the inflammatory response. ADP-ribosylation also regulates signaling and gene expression either on the cell surface (ARTCs), in the cytoplasm and in the nucleus (ARTDs) influencing transcription factor or pro-inflammatory gene expression. Thus, ADP-ribosylation induces and enhances inflammatory responses at multiple levels and PARPi may prevent the progression towards detrimental inflammation. 


\section{Box 3. The different phases of the immune responses}

Inflammation is orchestrated by different cell types that integrate diverse stimuli for the generation of a specific immune response to kill invading pathogens and/or to clear and regenerate damaged tissue ${ }^{109}$. A typical immune response can be divided in three phases: a recognition and initiation phase, a second amplification and modulation phase followed by a third effector phase (Figure below). Whereas the innate immune system senses and initiates inflammation (first phase), both innate and adaptive immune systems regulate the second and third phase ${ }^{32}$. The binding of PAMPs/DAMPS to different PRRs communicates the origin of the antigens recognized and the type of infection encountered to the immune cells, and provides the information which effector class of the immune response to raise. How the immune system interprets these signals is not yet fully understood and depends very much on the molecular patterns themselves. When focusing on the regulatory role of ADP-ribosylation during these three phases it not only important to define the involved cell types, but also to consider the expression of the enzymes (i.e. writer) involved in ADP-ribosylation (Figure below). Not all possible ADPribosylation writers are expressed to a detectable level in all cell types involved in the three phases (Figure below). This differential expression pattern will most likely facilitate future efforts to elucidate the role of ADP-ribosylation in these cells.

a)

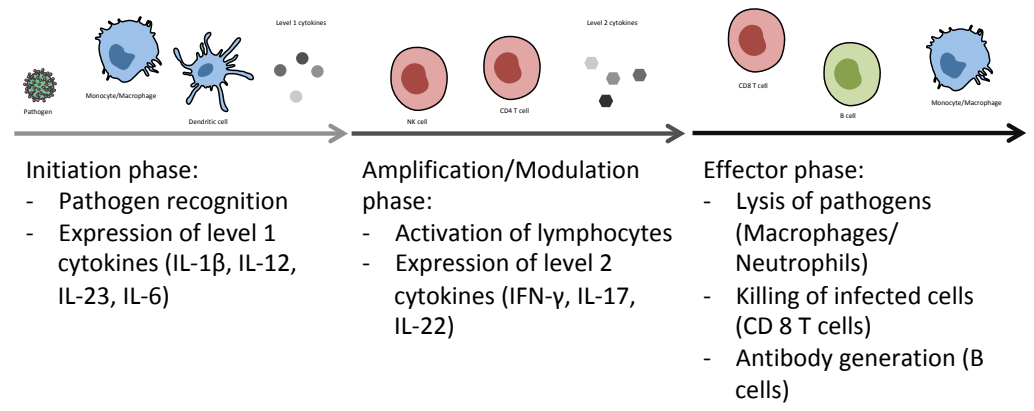

b)

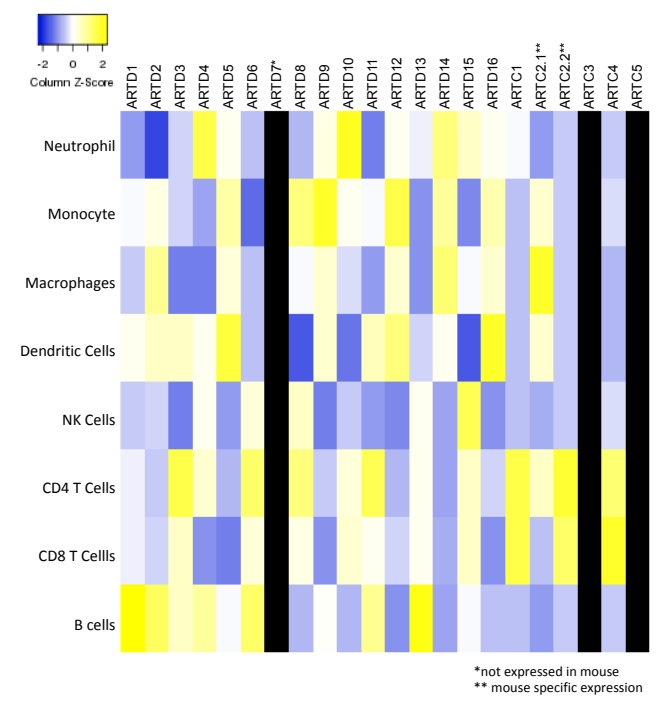

a) The immune response. Immune responses are orchestrated by specialized cell types in three distinct phases. Cells of the innate immunity sense PAMPs or DAMPs and initiate the first phase by level 1 cytokines. The activation of lymphocytes characterizes the second phase of an immune response and level 2 cytokines determine the third phase: effector functions and elimination of the pathogen.

b) Gene expression profiles of ADP-ribosylation writers in murine innate and adaptive immune cells. The microarray expression data (extracted from https://www.immgen.org) of ARTDs and ARTCs is displayed. 


\section{Outstanding Question box}

- Which are the tissue- and cell type-specific roles of individual ARTD family members and erasers during inflammation in vivo?

- Does $\mathrm{NAD}^{+}$availability and the energy status of a cell influence ADP-ribosylation during inflammation, such as macrophage polarization?

- Can ADP-ribosylation affect epigenetic memory of immune cells including immune training and tolerance?

- How do PARPi influence the immune system during cancer development?

- What are the ADP-ribosylation target proteins during inflammation and does ADPribosylation regulate their function? 


\section{Table 1. Ant-inflammatory effects of PARPi}

\begin{tabular}{|c|c|c|c|c|}
\hline Disease Model & Disease & Phenotype & Mechanism & Reference \\
\hline Hemorrhagic shock/Septic peritonitis & Septic shock & Inflammatory burden $\downarrow$ & Parthanatos $\downarrow$ & 140,141 \\
\hline \multirow[t]{2}{*}{ Collagen-induced arthritis } & Rheumatoid arthritis & Incidence/severity $\downarrow$ & n.d. & $142-144$ \\
\hline & & $\begin{array}{l}\text { Improved histological status in the knee and } \\
\text { paw } \\
\text { TNFa, IL-6, IL-12, IFNg, MIP2, IL-1b } \downarrow \text { in } \\
\text { the joints } \\
\text { IL-6, RANTES, MMP-3, IL-8, MCP-1 } \downarrow \text { in } \\
\text { fibroblast-like synoviocytes }\end{array}$ & NF- $\kappa \mathrm{B}$ and AP-1 binding $\downarrow$ & \\
\hline Streptozotocin-induced diabetes & Diabetes Type I & $\beta$-cell death $\downarrow$ & Parthanatos $\downarrow$ & 145 \\
\hline Myocardial ischemia-reperfusion & Myocardial infarction & Infarct size $\downarrow$ & n.d. & 146 \\
\hline & & $\begin{array}{l}\text { IL- } 6 \text { and CRP } \downarrow \text { in patients with myocardial } \\
\text { infarction }\end{array}$ & & 147 \\
\hline Il-10 knock-out mice & $\begin{array}{l}\text { Inflammatory bowel } \\
\text { disease }\end{array}$ & $\begin{array}{l}\text { Resistance in hapten-induced colitis rodent } \\
\text { models }\end{array}$ & Inflammatory cytokine expression $\downarrow$ & $141,148-151$ \\
\hline MPTP mouse model & Parkinson's disease & Reduced neuronal excitotoxicity & Parthanatos $\downarrow$ & 152 \\
\hline
\end{tabular}

Arrow down indicates down-regulation/reduction. n.d. $=$ not defined . 


\section{References:}

1. Bianchi, M. E. DAMPs, PAMPs and alarmins: all we need to know about danger. J Leukoc Biol 81, 1-5 (2007).

2. Hassa, P. O. \& Hottiger, M. O. The diverse biological roles of mammalian PARPs, a small but powerful family of poly-ADP-ribose polymerases. Front Biosci (2008).

3. Potaman, V. N., Shlyakhtenko, L. S., Oussatcheva, E. A., Lyubchenko, Y. L. \& Soldatenkov, V. A. Specific binding of poly(ADP-ribose) polymerase-1 to cruciform hairpins. J Mol Biol 348, 609-615 (2005).

4. Lonskaya, I. et al. Regulation of poly(ADP-ribose) polymerase-1 by DNA structure-specific binding. J. Biol. Chem. 280, 17076-17083 (2005).

5. Soldatenkov, V. A., Vetcher, A. A., Duka, T. \& Ladame, S. First evidence of a functional interaction between DNA quadruplexes and poly(ADP-ribose) polymerase-1. ACS Chem Biol 3, 214-219 (2008).

6. Ali, A. A. E. et al. The zinc-finger domains of PARP1 cooperate to recognize DNA strand breaks. Nat Struct Mol Biol 19, 685-692 (2012).

7. Robert, I., Karicheva, O., Reina San Martin, B., Schreiber, V. \& Dantzer, F. Functional aspects of PARylation in induced and programmed DNA repair processes: preserving genome integrity and modulating physiological events. Mol. Asp. Med. 34, 1138-1152 (2013).

8. De Vos, M., Schreiber, V. \& Dantzer, F. The diverse roles and clinical relevance of PARPs in DNA damage repair: current state of the art. Biochem Pharmacol 84, 137-146 (2012).

9. Ray Chaudhuri, A. \& Nussenzweig, A. The multifaceted roles of PARP1 in DNA repair and chromatin remodelling. Nat Rev Mol Cell Biol 18, 610-621 (2017).

10. Curtin, N. J. \& Szabó, C. Therapeutic applications of PARP inhibitors: Anticancer therapy and beyond. Mol. Asp. Med. 34, 1217-1256 (2013).

11. Lazebnik, Y. A., Kaufmann, S. H., Desnoyers, S., Poirier, G. G. \& Earnshaw, W. C. Cleavage of poly(ADP-ribose) polymerase by a proteinase with properties like ICE. Nature 371, 346-347 (1994).

12. Virág, L. \& Szabó, C. The therapeutic potential of poly(ADP-ribose) polymerase inhibitors. Pharmacol Rev 54, 375-429 (2002).

13. Pétrilli, V. et al. Noncleavable poly(ADP-ribose) polymerase-1 regulates the inflammation response in mice. J Clin Invest 114, 1072-1081 (2004).

14. Simbulan-Rosenthal, C. M., Rosenthal, D. S., Ding, R., Bhatia, K. \& Smulson, M. E. Prolongation of the 553 response to DNA strand breaks in cells depleted of PARP by antisense RNA expression. Biol Chem 253, 864-868 (1998).

15. Houtkooper, R. H., Cantó, C., Wanders, R. J. \& Auwerx, J. The secret life of NAD+: an old metabolite controlling new metabolic signaling pathways. Endocr Rev 31, 194-223 (2010).

16. Skidmore, C. J. et al. The involvement of poly(ADP-ribose) polymerase in the degradation of NAD caused by gamma-radiation and N-methyl-N-nitrosourea. Eur J Biochem 101, 135-142 (1979).

17. Goodwin, P. M., Lewis, P. J., Davies, M. I., Skidmore, C. J. \& Shall, S. The effect of gamma radiation and neocarzinostatin on NAD and ATP levels in mouse leukaemia cells. Biochim Biophys Acta 543, 576-582 (1978).

18. Bai, P. et al. PARP-1 Inhibition Increases Mitochondrial Metabolism through SIRT1 Activation. Cell Metabolism 13, 461-468 (2011).

19. Luo, X. \& Kraus, W. L. On PAR with PARP: cellular stress signaling through poly(ADPribose) and PARP-1. Genes Dev. 26, 417-432 (2012).

20. Bürkle, A. \& Virág, L. Poly(ADP-ribose): PARadigms and PARadoxes. Mol. Asp. Med. 34, 1046-1065 (2013).

21. Goto, S. et al. Poly(ADP-ribose) polymerase impairs early and long-term experimental stroke recovery. Stroke 33, 1101-1106 (2002).

22. Zhou, Y., Feng, X. \& Koh, D. W. Activation of cell death mediated by apoptosis-inducing factor due to the absence of poly(ADP-ribose) glycohydrolase. Biochemistry 50, 2850-2859 (2011).

23. David, K. K., Andrabi, S. A., Dawson, T. M. \& Dawson, V. L. Parthanatos, a messenger of death. Microbiol Mol Biol Rev 14, 1116-1128 (2009).

24. Galluzzi, L. et al. Molecular definitions of cell death subroutines: recommendations of the Nomenclature Committee on Cell Death 2012. Cell Death Differ 19, 107-120 (2012). 
25. Andrabi, S. A. et al. Poly(ADP-ribose) (PAR) polymer is a death signal. PNAS 103, 1830818313 (2006).

26. Yu, S.-W. et al. Apoptosis-inducing factor mediates poly(ADP-ribose) (PAR) polymerinduced cell death. PNAS 103, 18314-18319 (2006).

27. Wang, Y. et al. Poly(ADP-ribose) (PAR) binding to apoptosis-inducing factor is critical for PAR polymerase-1-dependent cell death (parthanatos). Sci Signal 4, ra20-ra20 (2011).

28. Kono, H. \& Rock, K. L. How dying cells alert the immune system to danger. Nat Rev Immunol 8, 279-289 (2008).

29. Yang, M. et al. Poly-ADP-ribosylation of HMGB1 regulates TNFSF10/TRAIL resistance through autophagy. Autophagy 11, 214-224 (2015).

30. Ditsworth, D., Zong, W.-X. \& Thompson, C. B. Activation of poly(ADP)-ribose polymerase (PARP-1) induces release of the pro-inflammatory mediator HMGB1 from the nucleus. $J$. Biol. Chem. 282, 17845-17854 (2007).

31. Yang, Z. et al. PARP-1 mediates LPS-induced HMGB1 release by macrophages through regulation of HMGB1 acetylation. J Immunol 193, 6114-6123 (2014).

32. Medzhitov, R. Origin and physiological roles of inflammation. Nature 454, 428-435 (2008).

33. Gupte, R., Liu, Z. \& Kraus, W. L. PARPs and ADP-ribosylation: recent advances linking molecular functions to biological outcomes. Genes Dev. 31, 101-126 (2017).

34. Hassa, P. O., Buerki, C., Lombardi, C., Imhof, R. \& Hottiger, M. O. Transcriptional coactivation of nuclear factor-kappaB-dependent gene expression by $\mathrm{p} 300$ is regulated by poly(ADP)-ribose polymerase-1. J. Biol. Chem. 278, 45145-45153 (2003).

35. Hassa, P. O. et al. Acetylation of poly(ADP-ribose) polymerase-1 by $\mathrm{p} 300 /$ CREB-binding protein regulates coactivation of NF-kappaB-dependent transcription. J. Biol. Chem. 280, 40450-40464 (2005).

36. Hassa, P. O., Covic, M., Hasan, S., Imhof, R. \& Hottiger, M. O. The enzymatic and DNA binding activity of PARP-1 are not required for NF-kappa B coactivator function. J. Biol. Chem. 276, 45588-45597 (2001).

37. Hassa, P. O. \& Hottiger, M. O. A role of poly (ADP-ribose) polymerase in NF-kappaB transcriptional activation. Biol Chem 380, 953-959 (1999).

38. Martinez-Zamudio, R. \& Ha, H. C. Histone ADP-ribosylation facilitates gene transcription by directly remodeling nucleosomes. Mol Cell Biol 32, 2490-2502 (2012).

39. Martínez-Zamudio, R. I. \& Ha, H. C. PARP1 enhances inflammatory cytokine expression by alteration of promoter chromatin structure in microglia. Brain Behav. 4, 552-565 (2014).

40. Oliver, F. J. et al. Resistance to endotoxic shock as a consequence of defective NF-kappaB activation in poly (ADP-ribose) polymerase-1 deficient mice. EMBO J 18, 4446-4454 (1999).

41. Minotti, R., Andersson, A. \& Hottiger, M. O. ARTD1 Suppresses Interleukin 6 Expression by Repressing MLL1-Dependent Histone H3 Trimethylation. Mol Cell Biol 35, 3189-3199 (2015).

42. Hottiger, M. O. SnapShot: ADP-Ribosylation Signaling. Molecular Cell 58, $-1134 . e 1$ (2015).

43. Krishnakumar, R. \& Kraus, W. L. The PARP Side of the Nucleus: Molecular Actions, Physiological Outcomes, and Clinical Targets. Molecular Cell 39, 8-24 (2010).

44. Rouleau, M., Aubin, R. A. \& Poirier, G. G. Poly(ADP-ribosyl)ated chromatin domains: access granted. J Cell Sci 117, 815-825 (2004).

45. Althaus, F. R. et al. Histone shuttling by poly ADP-ribosylation. Mol Cell Biochem 138, 5359 (1994).

46. Erener, S. et al. Inflammasome-Activated Caspase 7 Cleaves PARP1 to Enhance the Expression of a Subset of NF-\&kappa;B Target Genes. Molecular Cell 1-12 (2012). doi:10.1016/j.molcel.2012.02.016

47. Hassa, P. O. \& Hottiger, M. O. The functional role of poly(ADP-ribose)polymerase 1 as novel coactivator of NF-kappaB in inflammatory disorders. Cell Mol Life Sci 59, 1534-1553 (2002).

48. Carrillo, A. et al. Transcription regulation of TNF- $\alpha$-early response genes by poly(ADPribose) polymerase-1 in murine heart endothelial cells. Nucleic Acids Research 32, 757-766 (2004).

49. Zerfaoui, M. et al. Nuclear translocation of p65 NF-kappaB is sufficient for VCAM-1, but not ICAM-1, expression in TNF-stimulated smooth muscle cells: Differential requirement for PARP-1 expression and interaction. Cell Signal 20, 186-194 (2008).

50. Ha, H. C., Hester, L. D. \& Snyder, S. H. Poly(ADP-ribose) polymerase-1 dependence of 
stress-induced transcription factors and associated gene expression in glia. PNAS 99, 32703275 (2002).

51. Hottiger, M. O. Nuclear ADP-Ribosylation and Its Role in Chromatin Plasticity, Cell Differentiation, and Epigenetics. Ann Rev Biochem 84, 227-263 (2015).

52. Ke, Y. et al. PARP1 promotes gene expression at the post-transcriptiona level by modulating the RNA-binding protein HuR. Nature Comm. 8, 14632 (2017).

53. Ji, Y. \& Tulin, A. V. Post-transcriptional regulation by poly(ADP-ribosyl)ation of the RNAbinding proteins. Int J Mol Sci 14, 16168-16183 (2013).

54. Galbis-Martínez, M., Saenz, L., Ramírez, P., Parrilla, P. \& Yélamos, J. Poly(ADP-ribose) polymerase-1 modulates interferon-gamma-inducible protein (IP)-10 expression in murine embryonic fibroblasts by stabilizing IP-10 mRNA. Mol. Immunol. 47, 1492-1499 (2010).

55. Grossman, Z. \& Paul, W. E. Dynamic tuning of lymphocytes: physiological basis, mechanisms, and function. Ann Rev Immunol 33, 677-713 (2015).

56. Yélamos, J. et al. PARP-2 deficiency affects the survival of CD4+CD8+ double-positive thymocytes. EMBO J 25, 4350-4360 (2006).

57. Navarro, J. et al. PARP-1/PARP-2 double deficiency in mouse T cells results in faulty immune responses and T lymphomas. Sci Rep. 7, 41962 (2017).

58. Huang, J., Meyer, C. \& Zhu, C. T cell antigen recognition at the cell membrane. Mol. Immunol. 52, 155-164 (2012).

59. Adriouch, S. et al. ADP-ribosylation at R125 gates the $\mathrm{P} 2 \mathrm{X} 7$ ion channel by presenting a covalent ligand to its nucleotide binding site. FASEB J 22, 861-869 (2008).

60. Adriouch, S., Ohlrogge, W., Haag, F., Koch-Nolte, F. \& Seman, M. Rapid induction of naive $\mathrm{T}$ cell apoptosis by ecto-nicotinamide adenine dinucleotide: requirement for mono(ADPribosyl)transferase 2 and a downstream effector. J Immunol 167, 196-203 (2001).

61. Teege, S. et al. Tuning IL-2 signaling by ADP-ribosylation of CD25. Sci Rep. 5, 8959 (2015).

62. Okamoto, S., Azhipa, O., Yu, Y., Russo, E. \& Dennert, G. Expression of ADPribosyltransferase on normal $\mathrm{T}$ lymphocytes and effects of nicotinamide adenine dinucleotide on their function. J Immunol 160, 4190-4198 (1998).

63. Bluestone, J. A., Mackay, C. R., O'Shea, J. J. \& Stockinger, B. The functional plasticity of T cell subsets. Nature 9, 811-816 (2009).

64. Shevach, E. M. Certified professionals: CD4(+)CD25(+) suppressor T cells. $J$ Exp Med 193, F41-6 (2001).

65. Fontenot, J. D. \& Rudensky, A. Y. A well adapted regulatory contrivance: regulatory T cell development and the forkhead family transcription factor Foxp3. Nat Immunol 6, 331-337 (2005).

66. Zhang, P. et al. PARP-1 regulates expression of TGF- $\beta$ receptors in T cells. Blood 122, 2224-2232 (2013).

67. Nasta, F., Laudisi, F., Sambucci, M., Rosado, M. M. \& Pioli, C. Increased Foxp3+ regulatory $\mathrm{T}$ cells in poly(ADP-Ribose) polymerase-1 deficiency. J Immunol 184, 3470-3477 (2010).

68. Zhang, P. et al. PARP-1 controls immunosuppressive function of regulatory T cells by destabilizing Foxp3. PLOS One 8, e71590 (2013).

69. Macian, F. NFAT proteins: key regulators of T-cell development and function. Nat Rev Immunol 5, 472-484 (2005).

70. Valdor, R. et al. Regulation of NFAT by poly(ADP-ribose) polymerase activity in T cells. Mol. Immunol. 45, 1863-1871 (2008).

71. Olabisi, O. A. et al. Regulation of transcription factor NFAT by ADP-ribosylation. Mol Cell Biol 28, 2860-2871 (2008).

72. Saenz, L. et al. Transcriptional regulation by poly(ADP-ribose) polymerase-1 during T cell activation. BMC Genomics 9, 171 (2008).

73. Toller, I. M., Altmeyer, M., Kohler, E., Hottiger, M. O. \& Müller, A. Inhibition of ADP ribosylation prevents and cures helicobacter-induced gastric preneoplasia. Cancer Res 70, 5912-5922 (2010).

74. Iwasaki, A. \& Medzhitov, R. Control of adaptive immunity by the innate immune system. Nat Immunol 16, 343-353 (2015).

75. Barry, M. \& Bleackley, R. C. Cytotoxic T lymphocytes: all roads lead to death. Nat Rev Immunol 2, 401-409 (2002).

76. Jagtap, P. \& Szabó, C. Poly(ADP-ribose) polymerase and the therapeutic effects of its inhibitors. Nat Rev Drug Discov 4, 421-440 (2005).

77. Nathan, C. Points of control in inflammation. Nature 420, 846-852 (2002). 
78. Iwata, H. et al. PARP9 and PARP14 cross-regulate macrophage activation via STAT1 ADPribosylation. Nature Comm. 7, 12849 (2016).

79. Verheugd, P., Forst, A. H., Milke, L. \& Herzog, N. Regulation of NF- $\kappa B$ signalling by the mono-ADP-ribosyltransferase ARTD10. Nature Comm. (2013).

80. Kolaczkowska, E. \& Kubes, P. Neutrophil recruitment and function in health and inflammation. Nat Rev Immunol 13, 159-175 (2013).

81. Kefalas, P., Saxty, B., Yadollahi-Farsani, M. \& MacDermot, J. Chemotaxin-dependent translocation of immunoreactive ADP-ribosyltransferase-1 to the surface of human neutrophil polymorphs. Eur J Biochem 259, 866-871 (1999).

82. Grahnert, A. et al. Review: NAD +: a modulator of immune functions. Innate Immun 17, 212-233 (2011).

83. Paone, G. et al. ADP ribosylation of human neutrophil peptide-1 regulates its biological properties. PNAS 99, 8231-8235 (2002).

84. Paone, G. et al. ADP-ribosyltransferase-specific modification of human neutrophil peptide-1. J. Biol. Chem. 281, 17054-17060 (2006).

85. Rissiek, B. et al. Ecto-ADP-ribosyltransferase ARTC2.1 functionally modulates Fc $\gamma$ R1 and FcyR2B on murine microglia. Sci Rep. 7, 16477 (2017).

86. Moser, M. \& Leo, O. Key concepts in immunology. Vaccine 28 Suppl 3, C2-13 (2010).

87. Ambrose, H. E. et al. Poly(ADP-ribose) polymerase-1 (Parp-1)-deficient mice demonstrate abnormal antibody responses. Immunology 127, 178-186 (2009).

88. Mehrotra, P. et al. PARP-14 Functions as a Transcriptional Switch for Stat6-dependent Gene Activation. J. Biol. Chem. 286, 1767-1776 (2011).

89. Verheugd, P., Bütepage, M., Eckei, L. \& Lüscher, B. Players in ADP-ribosylation: Readers and Erasers. Curr. Protein Pept. Sci. 17, 654-667 (2016).

90. Min, W. \& Wang, Z.-Q. Poly (ADP-ribose) glycohydrolase (PARG) and its therapeutic potential. Front Biosci 14, 1619-1626 (2009).

91. Genovese, T. et al. Treatment with a novel poly(ADP-ribose) glycohydrolase inhibitor reduces development of septic shock-like syndrome induced by zymosan in mice. Crit Care Med 32, 1365-1374 (2004).

92. Cortes, U. et al. Depletion of the 110-kilodalton isoform of poly(ADP-ribose) glycohydrolase increases sensitivity to genotoxic and endotoxic stress in mice. Mol Cell Biol 24, 7163-7178 (2004).

93. Cuzzocrea, S. et al. PARG activity mediates intestinal injury induced by splanchnic artery occlusion and reperfusion. FASEB J 19, 558-566 (2005).

94. Lu, X.-C. M. et al. Post-treatment with a novel PARG inhibitor reduces infarct in cerebral ischemia in the rat. Brain Res 978, 99-103 (2003).

95. Patel, N. S. A. et al. Mice lacking the 110-kD isoform of poly(ADP-ribose) glycohydrolase are protected against renal ischemia/reperfusion injury. J Am Soc Nephrol 16, 712-719 (2005).

96. Cuzzocrea, S. et al. Role of poly(ADP-ribose) glycohydrolase in the development of inflammatory bowel disease in mice. Free Radical Biol Med 42, 90-105 (2007).

97. Atasheva, S., Frolova, E. I. \& Frolov, I. Interferon-Stimulated Poly(ADP-Ribose) Polymerases Are Potent Inhibitors of Cellular Translation and Virus Replication. J Virol. 88, 2116-2130 (2014).

98. Zhang, Y. et al. PARP9-DTX3L ubiquitin ligase targets host histone H2BJ and viral 3C protease to enhance interferon signaling and control viral infection. Nat Immunol (2015). doi:10.1038/ni.3279

99. Gao, G., Guo, X. \& Goff, S. P. Inhibition of retroviral RNA production by ZAP, a CCCHtype zinc finger protein. Science 297, 1703-1706 (2002).

100. Leung, A., Todorova, T., Ando, Y.\& Chang, P. Poly(ADP-ribose) regulates posttranscriptional gene regulation in the cytoplasm. RNA Biol 9, 542-548 (2012).

101. Seo, G. J. et al. Reciprocal inhibition between intracellular antiviral signaling and the RNAi machinery in mammalian cells. Cell Host Microbe 14, 435-445 (2013).

102. Karlberg, T. et al. Structural basis for lack of ADP-ribosyltransferase activity in poly(ADPribose) polymerase-13/zinc finger antiviral protein. J. Biol. Chem. 290, 7336-7344 (2015).

103. Kozaki, T. et al. Mitochondrial damage elicits a TCDD-inducible poly(ADP-ribose) polymerase-mediated antiviral response. PNAS 114, 2681-2686 (2017).

104. Li, L. et al. PARP12 suppresses Zika virus infection through PARP-dependent degradation of NS1 and NS3 viral proteins. Sci Signal 11, (2018).

105. Riffell, J. L., Lord, C. J. \& Ashworth, A. Tankyrase-targeted therapeutics: expanding 
opportunities in the PARP family. Nat Rev Drug Discov 11, 923-936 (2012).

106. Li, Z. et al. Herpes simplex virus requires poly(ADP-ribose) polymerase activity for efficient replication and induces extracellular signal-related kinase-dependent phosphorylation and ICP0-dependent nuclear localization of tankyrase 1. J Virol. 86, 492-503 (2012).

107. Murray, P. J. Macrophage Polarization. Annu. Rev. Physiol. 79, 541-566 (2017).

108. Murray, P. J. et al. Macrophage activation and polarization: nomenclature and experimental guidelines. Immunity 41, 14-20 (2014).

109. Abplanalp, J. \& Hottiger, M. O. Cell fate regulation by chromatin ADP-ribosylation. Semin. Cell Dev. Biol. 63, 114-122 (2017).

110. Simon, N. C., Aktories, K. \& Barbieri, J. T. Novel bacterial ADP-ribosylating toxins: structure and function. Nat Rev Microbiol 12, 599-611 (2014).

111. Hottiger, M. O., Hassa, P. O., Lüscher, B., Schüler, H. \& Koch-Nolte, F. Toward a unified nomenclature for mammalian ADP-ribosyltransferases. Trends Biochem Sci 35, 208-219 (2010).

112. Seman, M., Adriouch, S., Haag, F. \& Koch-Nolte, F. Ecto-ADP-ribosyltransferases (ARTs): emerging actors in cell communication and signaling. Curr. Med. Chem. 11, 857-872 (2004).

113. Teloni, F. \& Altmeyer, M. Readers of poly(ADP-ribose): designed to be fit for purpose. Nucleic Acids Research 44, 993-1006 (2016).

114. Meyer-Ficca, M. L., Meyer, R. G., Coyle, D. L., Jacobson, E. L. \& Jacobson, M. K. Human poly(ADP-ribose) glycohydrolase is expressed in alternative splice variants yielding isoforms that localize to different cell compartments. Exp. Cell Res. 297, 521-532 (2004).

115. Brochu, G. et al. Mode of action of poly(ADP-ribose) glycohydrolase. Biochim Biophys Acta 1219, 342-350 (1994).

116. Di Meglio, S. et al. Poly(ADPR) polymerase-1 and poly(ADPR) glycohydrolase level and distribution in differentiating rat germinal cells. Mol Cell Biochem 248, 85-91 (2003).

117. Gao, H. et al. Altered poly(ADP-ribose) metabolism impairs cellular responses to genotoxic stress in a hypomorphic mutant of poly(ADP-ribose) glycohydrolase. Exp. Cell Res. 313, 984-996 (2007).

118. Brochu, G., Shah, G. M. \& Poirier, G. G. Purification of poly(ADP-ribose) glycohydrolase and detection of its isoforms by a zymogram following one- or two-dimensional electrophoresis. Anal Biochem 218, 265-272 (1994).

119. Meyer, R. G., Meyer-Ficca, M. L., Whatcott, C. J., Jacobson, E. L. \& Jacobson, M. K. Two small enzyme isoforms mediate mammalian mitochondrial poly(ADP-ribose) glycohydrolase (PARG) activity. Exp. Cell Res. 313, 2920-2936 (2007).

120. Karlberg, T., Langelier, M.-F., Pascal, J. M. \& Schüler, H. Structural biology of the writers, readers, and erasers in mono- and poly(ADP-ribose) mediated signaling. Mol. Aspects Med. 34, 1088-1108 (2013).

121. Abplanalp, J. et al. Proteomic analyses identify ARH3 as a serine mono-ADPribosylhydrolase. Nat Commun 8, 2055 (2017).

122. Fontana, P. et al. Serine ADP-ribosylation reversal by the hydrolase ARH3. Elife 6, (2017).

123. Sharifi, R. et al. Deficiency of terminal ADP-ribose protein glycohydrolase TARG1/C6orf130 in neurodegenerative disease. EMBO J 32, 1225-1237 (2013).

124. Rosenthal, F. et al. Macrodomain-containing proteins are new mono-ADP-ribosylhydrolases. Nat Struct Mol Biol 20, 502-507 (2013).

125. Jankevicius, G. et al. A family of macrodomain proteins reverses cellular mono-ADPribosylation. Nat Struct Mol Biol 20, 508-514 (2013).

126. Altmeyer, M. \& Hottiger, M. O. Poly (ADP-ribose) polymerase 1 at the crossroad of metabolic stress and inflammation in aging. Aging (2009).

127. Kraus, W. L. \& Lis, J. T. PARP goes transcription. Cell 113, 677-683 (2003).

128. Kraus, W. L. PARPs and ADP-Ribosylation: 50 Years ... and Counting. Molecular Cell 58, 902-910 (2015).

129. Chen, J.-K., Lin, W.-L., Chen, Z. \& Liu, H.-W. PARP-1-dependent recruitment of coldinducible RNA-binding protein promotes double-strand break repair and genome stability. PNAS 115, E1759-E1768 (2018).

130. Lord, C. J. \& Ashworth, A. PARP inhibitors: Synthetic lethality in the clinic. Science 355, 1152-1158 (2017).

131. Shen, Y. et al. BMN673, a Novel and Highly Potent PARP1/2 Inhibitor for the Treatment of Human Cancers with DNA Repair Deficiency. Clin Cancer Res 19, 5003-5015 (2013).

132. Lord, C. J. \& Ashworth, A. Mechanisms of resistance to therapies targeting BRCA-mutant cancers. Nat Med 19, 1381-1388 (2013). 
133. Farmer, H. et al. Targeting the DNA repair defect in BRCA mutant cells as a therapeutic strategy. Nature 434, 917-921 (2005).

134. Bryant, H. E. et al. Specific killing of BRCA2-deficient tumours with inhibitors of poly(ADP-ribose) polymerase. Nature 434, 913-917 (2005).

135. Murai, J. et al. Stereospecific PARP trapping by BMN 673 and comparison with olaparib and rucaparib. Mol. Cancer Ther. 13, 433-443 (2014).

136. Murai, J. et al. Trapping of PARP1 and PARP2 by Clinical PARP Inhibitors. Cancer Res 72, 5588-5599 (2012).

137. Pommier, Y., O'Connor, M. J. \& de Bono, J. Laying a trap to kill cancer cells: PARP inhibitors and their mechanisms of action. Sci Transl Med 8, 362ps17-362ps17 (2016).

138. Wahlberg, E. et al. Family-wide chemical profiling and structural analysis of PARP and tankyrase inhibitors. Nat Biotech 30, 283-288 (2012).

139. Hottiger, M. O. Poly (ADP-ribose) polymerase inhibitor therapeutic effect: are we just scratching the surface? Exp. Opinion Therap. Targets (2015).

140. Jagtap, P., Soriano, F. G., Virág, L. \& Liaudet, L. Novel phenanthridinone inhibitors of poly (adenosine 5'-diphosphate-ribose) synthetase: potent cytoprotective and antishock agents. Crit Care (2002).

141. Di Paola, R. et al. Treatment with PARP-1 inhibitors, GPI 15427 or GPI 16539, ameliorates intestinal damage in rat models of colitis and shock. Eur J Pharmacol 527, 163-171 (2005).

142. Szabó, C. et al. Protection against peroxynitrite-induced fibroblast injury and arthritis development by inhibition of poly(ADP-ribose) synthase. PNAS 95, 3867-3872 (1998).

143. Gonzalez-Rey, E. et al. Therapeutic Effect of a Poly(ADP-Ribose) Polymerase-1 Inhibitor on Experimental Arthritis by Downregulating Inflammation and Th1 Response. PLOS One 2, e1071 (2007).

144. García, S., Bodaño, A., Pablos, J. L., Gómez-Reino, J. J. \& Conde, C. Poly(ADP-ribose) polymerase inhibition reduces tumor necrosis factor-induced inflammatory response in rheumatoid synovial fibroblasts. Ann Rheum Dis 67, 631-637 (2008).

145. Garcia Soriano F et al. Diabetic endothelial dysfunction: the role of poly(ADP-ribose) polymerase activation. Nat Med 7, 108-113 (2001).

146. Liaudet, L. et al. Suppression of poly (ADP-ribose) polymerase activation by 3aminobenzamide in a rat model of myocardial infarction: long-term morphological and functional consequences. Br J Pharmacol 133, 1424-1430 (2001).

147. Morrow, D. A. et al. A randomized, placebo-controlled trial to evaluate the tolerability, safety, pharmacokinetics, and pharmacodynamics of a potent inhibitor of poly(ADP-ribose) polymerase (INO-1001) in patients with ST-elevation myocardial infarction undergoing primary percutaneous coronary intervention: results of the TIMI 37 trial. $J$ Thromb Thrombolyssis 27, 359-364 (2009).

148. Jijon, H. B. et al. Inhibition of poly(ADP-ribose) polymerase attenuates inflammation in a model of chronic colitis. Am J Physiol Gastrointest Liver Phsiol 279, G641-51 (2000).

149. Mazzon, E. et al. GPI 6150, a PARP inhibitor, reduces the colon injury caused by dinitrobenzene sulfonic acid in the rat. Biochem Pharmacol 64, 327-337 (2002).

150. Sánchez-Fidalgo, S., Villegas, I., Martín, A., Sánchez-Hidalgo, M. \& Alarcón de la Lastra, C. PARP inhibition reduces acute colonic inflammation in rats. Eur J Pharmacol 563, 216-223 (2007).

151. Zingarelli, B., O'connor, M. \& Hake, P. W. Inhibitors of poly (ADP-ribose) polymerase modulate signal transduction pathways in colitis. Eur J Pharmacol 469, 183-194 (2003).

152. Wang, H., Shimoji, M., Yu, S.-W., Dawson, T. M. \& Dawson, V. L. Apoptosis inducing factor and PARP-mediated injury in the MPTP mouse model of Parkinson's disease. Ann N.Y. Acad Sci 991, 132-139 (2003). 